\title{
Berzelius Symposium 93: International Conference on Thrombosis and Embolism
}

\author{
Md. Shahidul Islam, MD, $\mathrm{PhD}^{1,2}$ Sam Schulman, MD, $\mathrm{PhD}^{3}$ \\ ${ }^{1}$ Department of Clinical Science and Education, Karolinska Institutet, \\ Stockholm, Sweden \\ 2 Department of Internal Medicine, Uppsala University Hospital, \\ Uppsala University, Uppsala, Sweden \\ ${ }^{3}$ Department of Medicine, Thrombosis and Atherosclerosis Research \\ Institute, McMaster University, Hamilton, Ontario, Canada
}

Semin Thromb Hemost 2017;43:811-813.

\section{Overview}

The Swedish Society of Medicine in collaboration with Karolinska Institutet organized the 93rd Berzelius Symposium "International Conference on Thrombosis and Embolism" in Stockholm, November 17-18, 2016. This international conference covered advances, breakthroughs, and many important aspects of thrombosis, embolism, and hemostasis, in the context of pathogenesis and treatment of diverse clinical conditions. The topics included intensive discussions on clinical conditions in different patient groups in challenging clinical situations.

\section{Background}

Jöns Jacob Berzelius, born in 1779, was a Swedish chemist, although he started off as a physician. He became famous for his work with assignment of weights to atoms, using oxygen as a reference with atomic weight set at 100 . Berzelius also assigned the $1+2$ letter abbreviations to the atomic names (O for oxygen, $\mathrm{Cu}$ for copper, etc.). In 1807, the Karolinska Institutet appointed him as professor in chemistry and pharmacy. In the honor of his name and under the auspices of the Swedish Society of Medicine, international symposia are frequently arranged to promote dissemination of medical science.

\section{Meeting Details}

The organizing committee consisted of Md. Shahidul Islam (chair), Sweden; Marika Bajc, Sweden; Alun H. Davis, United Kingdom; Søren Hess, Denmark; Paolo Prandoni, Italy; and Raveena Ravikumar, United Kingdom. The meeting was coordinated by Annie Melin of the Swedish Society of Medicine. The meeting took place at the society's beautiful building at Klara Östra Kyrkogata in central Stockholm.
Address for correspondence Md. Shahidul Islam, MD, PhD, Department of Clinical Science and Education, Karolinska Institutet, Södersjukhuset, Research Center, 3rd floor, 11883 Stockholm, Sweden (e-mail: shahidul.islam@ki.se).

The conference covered advances, breakthroughs, and important aspects of thrombosis and embolism in the context of pathogenesis and treatment of diverse medical conditions. The scope of the conference ranged from basic studies at cellular and molecular levels, through clinical manifestations and diagnostic considerations, to the treatment and follow-up of selected medical conditions. Diverse topics including clinical conditions in different patient groups and challenging clinical situations were covered. The conference was attended by junior researchers and physicians as well as by the experts, from scientists engaged in basic research to the bedside clinicians, from more than 10 different countries around the world.

Paolo Prandoni, Bengt Zöller, Wade S. Smith, Thomas Renné, Raveena, Ravikumar, Poul Henning Madsen, Christopher Kabrhel, Massimo Miniati, Sam Schulman, Jonathan Coutinho, Timothy R. Smith, David J. Cote, Vlad C. Radulescu, Christian Rylander, and Maria Boddi delivered the lectures or chaired the sessions. Some photos taken at the meeting are shown in - Fig. 1.

The titles of the topics included are as follows:

- Epidemiology of familial aggregation of venous thromboembolism.

- Atrial appendage ligation for the prevention of stroke from atrial fibrillation.

- Endovascular stroke therapy.

- Contact system of coagulation: new perspectives for safe anticoagulation.

- Whom to test for pulmonary embolism: view of a practicing respiratory physician.

- The value of pulmonary embolism response team.

- Pulmonary infarction: an often unrecognized clinical entity.

- Update on treatment of venous thromboembolism.

- Cerebral venous thrombosis.
Issue Theme Recent Advances in Thrombosis and Hemostasis-Part II; Guest Editors: Sam Schulman, MD, PhD, and Md. Shahidul Islam, MD, PhD.
Copyright (c 2017 by Thieme Medical Publishers, Inc., 333 Seventh Avenue, New York, NY 10001, USA. Tel: +1(212) 584-4662.
DOI https://doi.org/ 10.1055/s-0037-1605565. ISSN 0094-6176. 


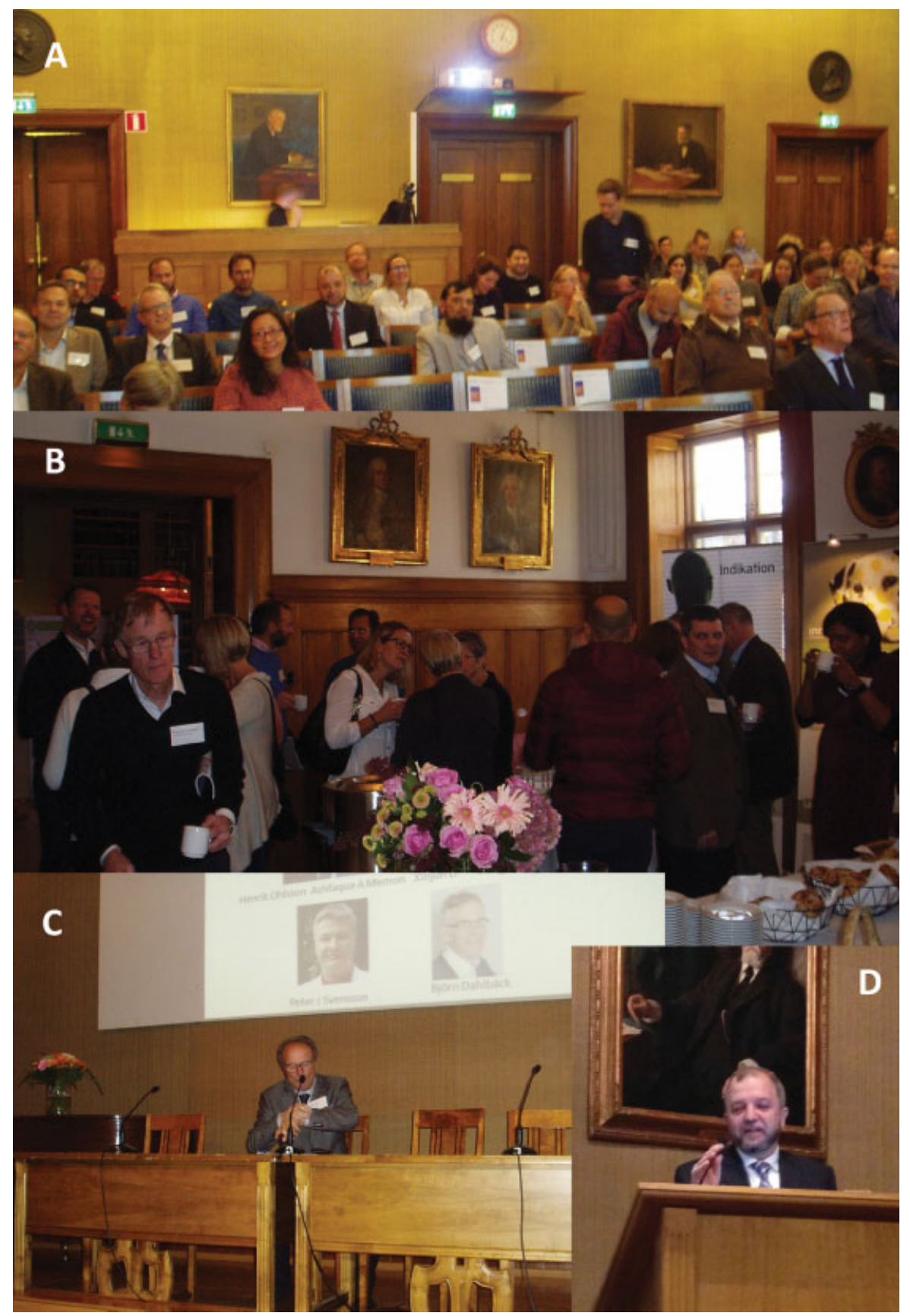

Fig. 1 Select photos from the 93rd Berzelius Symposium. (A) The audience and the venue, the Swedish Society of Medicine building at Klara Östra Kyrkogata in central Stockholm. (B) Discussions around thrombosis and hemostasis during a coffee break. (C) Paolo Prandoni, moderator, keeping track of the time. (D) Vlad Radulescu presenting on "Anticoagulation in children."

- High-grade glioma patients: treatment options for VTE prevention.

- Overview of VTE in patients undergoing craniotomy for brain tumors.

- Venous thromboembolism in children.

- Postthrombotic syndrome.

- Optimal duration of anticoagulation in patients with unprovoked venous thromboembolism.

- Catheter-directed thrombolysis and pharmacomechanical thrombolysis.

- Deep vein thrombosis in intensive care.
- Controversies in the management of venous thromboembolism in patients with cancer.

- Bleeding complications and management on anticoagulant therapy.

- Treatment of massive pulmonary embolism associated with hypotension: results from a newly introduced multidisciplinary standard operating procedure in Sweden.

The final session of the meeting was a panel discussion, facilitated by Sam Schulman, where the speakers and members of the audience contributed actively generating some new ideas for future research. 
Selected presentations from the meeting have been mirrored by articles recently published in two special issues of this journal, Seminars in Thrombosis \& Hemostasis, under the title of Advances in Thrombosis and Hemostasis. ${ }^{1,2}$

The social programs during the meeting included a dinner for the faculty hosted by the Swedish Society of Medicine at the society's beautiful building, and a reception for all participants at the Stockholm City Hall, one of the Stockholm's most famous buildings, and the venue of the Nobel Prize banquet. The reception hosted by the Stockholm City Council and the Stockholm County Council started with a welcome speech from the president of the Stockholm County Council, Annika Sandström, followed by a speech of thanks from Md. Shahidul Islam. After these formalities, the guests were invited to mingle around the buffet dinner, which was followed by a guided tour of the City Hall. The social activities as well as generous breaks during the scientific program allowed for plenty of informal discussions on the presented topics and other related ideas.

\section{Acknowledgment}

The organizers of the meeting are grateful to the Editor-inChief of this journal, Emmanuel J. Favaloro, for his assistance in bringing to fruition the two related issues of Seminars in Thrombosis $\mathcal{E}$ Hemostasis, ${ }^{1,2}$ in part reflecting select contributions from presenters at the 93rd Berzelius Symposium.

\section{References}

1 Schulman S, Islam MS. Recent advances in thrombosis and hemostasis - Part I. Semin Thromb Hemost 2016;42(08):805-807

2 Schulman S, Islam MS. Recent advances in thrombosis and hemostasis - Part II. Semin Thromb Hemost 2017;43(08):809-810 